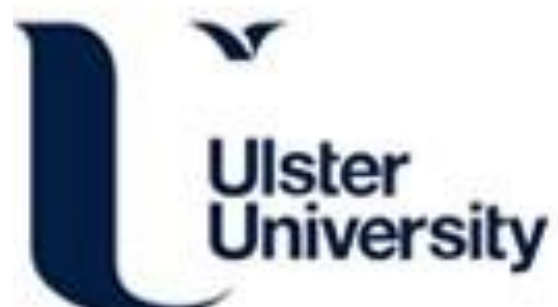

\section{Automatic feature selection of motor imagery EEG signals using differential evolution and learning automata}

Bhattacharyya, S., Sengupta, A., Chakraborti, T., Konar, A., \& Tibarewala, D. N. (2014). Automatic feature selection of motor imagery EEG signals using differential evolution and learning automata. MEDICAL \& BIOLOGICAL ENGINEERING \& COMPUTING, 52, 131-139. https://doi.org/10.1007/s11517-013-1123-9

Link to publication record in Ulster University Research Portal

Published in:

MEDICAL \& BIOLOGICAL ENGINEERING \& COMPUTING

Publication Status:

Published (in print/issue): 28/02/2014

DOI:

10.1007/s11517-013-1123-9

\section{Document Version}

Author Accepted version

\section{General rights}

Copyright for the publications made accessible via Ulster University's Research Portal is retained by the author(s) and / or other copyright owners and it is a condition of accessing these publications that users recognise and abide by the legal requirements associated with these rights.

\section{Take down policy}

The Research Portal is Ulster University's institutional repository that provides access to Ulster's research outputs. Every effort has been made to ensure that content in the Research Portal does not infringe any person's rights, or applicable UK laws. If you discover content in the Research Portal that you believe breaches copyright or violates any law, please contact pure-support@ulster.ac.uk. 


\section{Automatic Feature Selection of Motor Imagery EEG Signals using Differential Evolution and Learning Automata}

Saugat Bhattacharyya ${ }^{1}$, Abhronil Sengupta $^{1}$, Tathagatha Chakraborti ${ }^{1}$,

Amit Konar ${ }^{1}$, D.N.Tibarewala ${ }^{2}$

${ }^{1}$ Department of Electronics and Telecommunication Engineering, ${ }^{2}$ School of Bioscience and Engineering, Jadavpur University, Kolkata, India-700032

Corresponding Author:

Saugat Bhattacharyya,

e-mail: saugatbhattacharyya@gmail.com

tel: +919932866197

The total number of words of the manuscript, including entire text from title page to figure legends: $\mathbf{5 9 9 3}$

The number of words of the abstract: 177

The number of figures: $\mathbf{3}$

The number of tables: $\mathbf{5}$ 
Brain-Computer Interfacing has been the most researched technology in neuro-prosthesis in the last two decades. Feature extractors and classifiers play an important role in BCI research for the generation of suitable control signals to drive an assistive device. Due to the high dimensionality of feature vectors in practical BCI systems, implentation of efficient feature selection algorithms have been an integral area of research in the past decade. This article proposes an efficient feature selection technique, realized by means of an evolutionary algorithm, which attempts to overcome some of the shortcomings of several state of the art approaches in this field. The outlined scheme produces a subset of salient features which improves the classification accuracy while maintaining a trade-off with the computational speed of the complete scheme. For this purpose, an efficient memetic algorithm has also been proposed for the optimization purpose. Extensive experimental validations have been conducted on two real-world datasets to establish the efficacy of our approach. We have compared our approach to existing algorithms and have established the superiority of our algorithm to the rest.

Brain-Computer Interfacing, Feature Selection, Motor Imagery, Memetic Algorithm, Differential Evolution, Learning Automata, Power Spectral Density.

\section{Introduction}

It is well-known that intentions for any actions performed by a person originates from the brain [24, 25, 27]. Brain-computer Interfacing (BCI) extracts, decodes and translates these intentions into control commands to drive an external device for rehabilitative applications [3, 20, 30]. Other areas of application of BCI include robotics, communication, gaming and virtual reality [13, 15, 16, 33, 44]. Motor imagery (movement based) brain signals[12] is one of the most frequently researched field in BCI. For the acquisition of brain signals, both invasive and non-invasive means have been employed in BCI research which include electroencephalography (EEG), magnetoencephalography (MEG), electrocorticography (ECoG), intra-cortical electrodes and functional magnetic resonance imaging (fMRI) [28]. Among these, the EEG is preferred because it is non-invasive, easily available, portable and has very good temporal resolution $[12,33]$. The EEG signals during motor imagery experiments are acquired from C3 and C4 electrode locations[29] (based on the 10-20 electrode system) because they are directly placed above the motor cortex areas of the brain [19].

A general EEG-BCI module contains the following stages: pre-processing of the raw EEG, feature extraction from the EEG and classification of the EEG[23]. Here, time, frequency, time-frequency and non-linear signal processing methods are employed for feature extraction $[12,33,38-40]$ along with linear and non-linear methods as the classifiers [2, 32]. Sometimes, the features extracted from the EEG by the BCI have high dimensionality [22,32] which may result in two major drawbacks: (a) increase of the computational time of the classifier, and (b) EEG signals have poor signal-to-noise ratio 
[32] and are susceptible to the inclusion of features which behave as outliers and therefore reduce the classification accuracy.Thus during the past few decades researchers has included a feature selection stage before the classification stage [23]. This stage selects a subset of features from the original feature set having an enhanced discriminative power [40]. Some commonly used feature selection algorithms include Sequential Forward Floating Search [14], Sequential Forward Search [21], Principal Component Analysis [1], Singular Value Decomposition [17], Independent Component Analysis [7], Curvilinear Component Analysis, kernel Principal Component Analysis [34]. Existing approaches in feature selection suffer from few major drawbacks, which are: (a) Sometimes it is seen that even if the variances are good among components, they still have low classification performance. It may be due to the fact that the concerned algorithm failed to remove the redundant features. Determination and removal of redundant features is not possible simply by inspection of the feature set.

(b) Many of the popular feature extraction techniques perform a linear transformation of the original feature set to a vector of low dimensionality for consideration in the classifier stage. (c) The optimal number of reduced features to be considered in the classifier stage after dimensions reduction is determined by cumbersome experimental validations. The reduced features in most cases are a linear transformation of the original feature set. Thus even if the feature set used in the classifier stage is reduced we must still measure the original features. Here, we have solved the above problems by designing an algorithm to choose an optimal set of features from the original feature set itself. So all the features are not employed in the classification stage. Here, cumbersome experimental validations are avoided and a simple run of the optimizer is sufficient, which also optimizes the classifier performance.

The main contribution of this paper lies in the usage of evolutionary approach to the feature selection module, whose best $d$ features are selected from the total feature vector $\mathbf{D}$, where $d$ lies between $(0, \mathbf{D}]$. The algorithm is based on a population of members which are represented by two components, namely the Activation Thresholds and the Scale Factors. Activation Thresholds determine the corresponding features to be selected while the Scale Factors determine the amount by which a particular feature has to be scaled. On the basis of these two representations each population member undergoes the evolution process to produce fitter (with respect to the cost function) individuals. Another significant contribution of the paper is the application of our proposed memetic algorithm [35] to optimize the cost function. Memetic algorithms (MA) are population based search heuristics that integrate the mutual benefits of natural and cultural evolution.

Evolutionary algorithms (considering only genetic evolution) often get trapped in a local minima. Cultural Evolution, on the other hand, (on integration with genetic evolution) has 
proved to be more robust in this respect. This paper employs an intelligent optimization method which uses Differential Evolution (DE) [31, 37] as the genetic evolution tool and Learning Automata (LA) [26] for realizing the cultural evolution phase on two different motor imagery EEG datasets. Here, we have employed Power Spectral Estimates for feature extraction and Support Vector Machine for classification.

The rest of the paper is organized as follows. Section II describes our proposed framework of feature selection using DE and LA. Section III deals with the experiments undertaken to study the performance of our proposed approach. In section IV we compare our approach with other feature selection approaches. The concluding remarks are given in Section V.

\section{The Proposed Approach}

The proposed approach aims to reduce the dimensions of a feature vector based on a synergistic operation between an evolutionary algorithm and a supervised learning classifier. Here, number of trial vectors are formed with different number of features for the same data set which constructs a pseudo feature vector from the original dataset such that each data point consists only of the selected features. Precision of each possible combination of selected features is quantitatively evaluated with the classification accuracy obtained by testing the learning classifier. Then, through a mechanism of mutation and natural selection, eventually, the best solutions start dominating the population, whereas the bad ones are eliminated. Ultimately, the evolution of solutions converges when the fittest solution represents a near-optimal partitioning of the data set with respect to the employed validity index. In this way, the optimal number of features are selected using our proposed framework. Here, we have employed the use of LA-DE algorithm [35] and SVM for this purpose.

\subsection{The LA-DE Algorithm}

In the proposed framework, the global search mechanism is accomplished by successive generations of DE, while the optimal control parameters for individual members of the population in every generation is provided from a meme pool [43], for given scaling parameter $F$ which is maintained through the generations. A meme is defined as a unit of cultural information [43]. The meme selection process [43] is controlled by the state transition probability matrix $S_{i, j}$, where the row indices represent the states of the stochastic automata [26] and the column indices represent the actions performed by the automata at a particular state. The rows correspond to the population members ranked in the order of their decreasing fitness values and the columns 
correspond to uniform quantized values of the control parameter (i.e. $F$ ) in a given range, say $(0,2]$. In an evolutionary algorithm framework "fitness" signifies the performance of a population member with respect to a cost function (see section 2.3). The principles used in designing the LA-DE algorithm is outlined below.

\subsubsection{Initialization:}

LA-DE starts with a population of $N P D$-dimensional parameter vectors, each population vector representing a possible solution vector $\vec{Z}$. The population members are initialized according to a uniform random distribution along every dimension, within the prescribed minimum and maximum bounds: $\vec{Z}_{\text {min }}=\left\{z_{\text {min-1 }}, z_{\text {min-2 }}, \ldots, z_{\text {min }-D}\right\}$ and $\vec{Z}_{\text {max }}=\left\{z_{\max -1}, z_{\max -2}, \ldots, z_{\max -D}\right\}$. This ensures that for a reasonable number of vectors, the initial population covers the entire search space uniformly. Hence, we may initialize the $j^{\text {th }}$ component of the $i^{\text {th }}$ vector at generation $t=0$ as

$$
z_{i, j}(t)=z_{j-\min }+\operatorname{rand}_{i, j}(0,1) \times\left(z_{j-\max }-z_{j-\min }\right)
$$

The state transition probability matrix is initialized with equal values of 0.05 for 20 quantized levels of the parameter $F\left(F_{1}, F_{2}, \ldots, F_{20}\right)$. This is in accordance with the principle of unavailability of a priori information about the environment and assuming all actions to be equally likely at the initial stage.

\subsubsection{Adaptive Selection of Meme:}

The next step involves the selection of $F_{j}$ from the meme pool $\left(F_{1}, F_{2}, \ldots, F_{20}\right)$ (for a member of state $S_{i}$ ) such that the cumulative probability of selection of $F=F_{j}$ through $F_{j-1}$ is greater than a random number $r$ in the range $[0,1]$ for each population member, i.e.

$$
\sum_{m=1}^{j-1} p_{s_{i, m}}<r \leq \sum_{m=j}^{20} p_{s_{i, m}}
$$

where, $p_{s_{i, j}}$ is the state transition probability vector at state $S_{i}$. Here, Roulette Wheel Selection [26] has been used for the selection of potentially useful memes. This entails that fitter memes would have higher probabilities of selection, but the memes with poorer fitness also manage to survive and contribute some components in the course of evolution. Thus this selection mechanism ensures that the diversity of the meme population is effectively maintained. 


\subsubsection{Differential Evolution}

- Mutation (DE/current-to-best/1): First, DE generates a donor vector $\vec{V}_{i}(t)$ corresponding to each population member $\vec{Z}_{i}(t)$ by randomly selecting two other members $\vec{Z}_{\text {rand }-1}(t)$ and $\vec{Z}_{\text {rand }-2}(t)$, where

$$
\vec{V}_{i}(t)=\vec{Z}_{i}(t)+F\left(\vec{Z}_{\text {best }}(t)-\vec{Z}_{i}(t)\right)+F\left(Z_{\text {rand }-1}(t)-\vec{Z}_{\text {rand }-2}(t)\right)
$$

and $F$ is the scaling factor (selected from the meme pool adaptively) which is used for linear scaling of the difference vectors during the mutation operation and $\vec{Z}_{b e s t}(t)$ is the population member with the best fitness.

- Crossover: Following the generation of the donor vectors, crossover operation is performed to increase the potential diversity of the population. There are two types of crossover (recombination) schemes- binomial and exponential [6,9]. In the proposed realization we have used binomial crossover, where the D components are changed whenever a randomly generated number, in the range $[0,1]$ following a binomial distribution, is less than or equal to the crossover ratio. Here, a trial vector $\vec{U}_{i}(t)$ is generated for each pair of $\vec{V}_{i}(t)$ and $\vec{Z}_{i}(t)$ by (4).

$$
u_{i, j}(t)=\left\{\begin{array}{rr}
v_{i, j}(t), & \text { if } \text { rand }_{i, j} \leq \text { Cror } \\
z_{i, j}(t), & j=j_{\text {rand }}\left(j_{\text {rand }} \in[1, D]\right)
\end{array}\right.
$$

where $\operatorname{rand}_{i, j}(0,1)$ is a uniformly distributed random number lying in $[0,1]$ and $C r$ is the cross-over ratio.

- Selection: The next step of DE decides whether the trial vector $\vec{U}_{i}(t)$ or the target vector $\vec{Z}_{i}(t)$ is selected for the next generation, according to their fitness. The selection process is

$$
\begin{aligned}
\vec{Z}_{i}(t+1) & =\vec{U}_{i}(t) \text { if } f\left(\vec{U}_{i}(t)\right) \leq f\left(\vec{Z}_{i}(t)\right) \\
& =\vec{Z}_{i}(t) \text { if } f\left(\vec{U}_{i}(t)\right)>f\left(\vec{Z}_{i}(t)\right)
\end{aligned}
$$

where $f(\vec{x})$ is the fitness. 


\subsubsection{Update of state transition probability matrix}

Let a member at state $S_{m}$ on selection of $F_{j}$ from the meme pool produces a trial vector after mutation and crossover. If the fitness of the trial vector increases, then the state transition probabilities are updated according to (6), otherwise it is updated according to (7). Here, the linear reinforcement scheme[35], is employed for the updation process, which is

$$
\begin{aligned}
& \text { If } f\left(\vec{x}_{t}\right)>f\left(\vec{x}_{t-1}\right), \\
& \qquad\left\{\begin{array}{rrr}
p_{j}(t+1) & = & (1-a) \cdot p_{j}(t) \forall j \neq i \text { for state } S_{m} \\
p_{i}(t+1) & = & p_{i}(t)+a \cdot\left(1-p_{i}(t)\right)
\end{array}\right.
\end{aligned}
$$

Otherwise ,

$$
\left\{\begin{array}{rlr}
p_{j}(t+1) & =\frac{b}{e-1}+(1-b) \cdot p_{j}(t) \forall j \neq i \\
p_{i}(t+1) & = & (1-b) \cdot p_{i}(t)
\end{array}\right.
$$

where, $a \epsilon[0,1]$ is the reward response, $b \in[0,1]$ is the penalty response and $e$ is the number of actions of the automata process.

\subsubsection{State Assignment}

Following the updation of the state transition probability matrix, we sort the the population members in decreasing order of their fitness and assign their corresponding states.

\subsubsection{Convergence}

After each evolution, we repeat from step-2 (see section 2.5) until the termination conditions are satisfied. The algorithm is stopped if maximum number of generations (gen_max) is reached or the cost function falls below a predefined level.

The overall schematic of the proposed LA-DE algorithm for feature selection is shown in Fig. 1.

Figure 1.

\subsection{Solution Representation and Fitness Evaluation}

In order to judge the quality of the proposed feature selection method, we partition the entire data set into three mutually exclusive partitions $\phi 1, \phi 2$ and $\phi 3$ for training, validation and testing, respectively. $\phi 1$ is employed to train the classifier for each population member and $\phi 2$ is used for fitness function calculation. Finally the 
optimal set of selected features, obtained as a result of optimization, is tested on $\phi 3$. For this purpose, $\mathrm{k}$-fold cross-validation technique [2] is employed and the features are normalized in the range $[0,10]$ before partitioning to reduce ambiguity. If $C A$ is the classification accuracy for the validation set $\phi 2$, then the fitness of the $j^{\text {th }}$ population member will be given by

$$
f_{j}=1 / C A
$$

Thus, minimization of this cost equation ensures population members with better validation stage classification accuracy to be selected during the evolution phase.

For the total set of $D$ features, we represent each member of the population (of $N P$ members $)$ participating in evolution as a 2-dimensional vector $P_{j}=\left(w_{1 j}, \ldots, w_{2 p j}\right)$ where the $i^{\text {th }}$ component of the vector, $w_{i j}\left(w_{i j} \in[0,1], i=1,2, \ldots, D ; j=1,2, \ldots, N P\right)$ represents the Activation Thresholds for the respective features. We state that if a particular component is greater than 0.5 then the corresponding feature feat is considered for validation and testing in the classifier stage. On the other hand, the $j^{\text {th }}$ component of the vector, $w_{i j}\left(w_{i j} \in[0,1], i=D+1, D+2, \ldots, 2 D ; j=1,2, \ldots, N\right)$ represents the Scaling Factors for the respective features. The Activation Thresholds and Scaling Factors determine which features are to be included in the final set $S_{j}$ of optimal features. The selection for the $i^{\text {th }}$ feature and $j^{\text {th }}$ population member is made as follows:

$$
\begin{aligned}
& \text { If }\left(w_{i j}>0.5\right) \\
& \text { feat }_{i} \leftarrow w_{i+D_{j}} \times \text { feat }_{i} \\
& \text { feat }_{i} \in S_{j} \\
& \text { else } \\
& \quad \text { ignore feat }_{i}
\end{aligned}
$$

Thus an Activation Weight of greater than 0.5 selects a feature for training and validation, and in that case the feature is multiplied by the corresponding Scaling Factor. This is done to enhance the discriminating power of the classifier. Linear scaling of features before classification enables the classifier to discriminate more efficiently along the feature axis with bigger scale factors. In this way the weights not only determine which feature to select but also the importance of the selected features. As an example we consider the weight vector $P_{j}$ of the $j^{\text {th }}$ population member in Fig. 2. Taking $D=6$, i.e. a total of 6 features, the values of the weights shown indicate that the 1st, 2nd and 3rd features are to be selected for training and validation after multiplication with appropriate 
scaling factors while the rest are ignored. Thus the set of selected features for the $j^{\text {th }}$ population member becomes $S_{j} \equiv\left\{0.68 \times f_{1}, 0.76 \times f_{2}, 0.44 \times f_{3}\right\}$.

Figure 2.

\subsection{Final Feature Selection}

The optimization continues until a stipulated number of generations have reached or an acceptable rate of classification is obtained. After termination, the population member with the best fitness is selected for testing $\phi 3$. The weights of this vector determine the final selection of features. Once again the chosen features are subjected to linear scaling by their corresponding weights. This ensures that throughout the process of training, validation and testing the features are multiplied by the same scaling factors and hence there is no scope of any misrepresentation of data.

\subsection{Pseudo Code}

The pseudocode for the complete algorithm is given here.

Step I: Initialize a set of NP vectors each with $2 D$ components initialized randomly between 0 and 1 .

Step II: Select features according to rule (8) for every population member.

Step III: Train classifier on $\phi 1$ with selected feature set.

Step IV: Validate on $\phi 2$ and calculate fitness of population members.

Step V: Update population members according to the evolutionary algorithm guided by the fitness values calculated above.

Step VI: if $g e n<g^{2} n_{M A X}$ goto Step II, else select member with the best fitness to get the final set of features. Here gen $_{\text {MAX }}$ denotes the maximum number of generations.

\section{Experiments and Results}

This section provides details on the experiments undertaken to examine the performance of our proposed feature selection algorithm. For this purpose, the power spectral density (PSD) estimates of two separate motor imagery datasets are employed as features. These features are fed to our proposed LA-DE feature selection algorithm to reduce the dimensions of the feature vector. Support Vector Machines (SVM) is employed to optimize the results of the LA-DE and validate its performance from the recognition accuracy of the given EEG datasets. 


\subsection{Dataset I: Dataset IVa from BCI Competition III}

This dataset comprises EEG recordings of five healthy subjects (namely, $a a, a l, a v, a w$ and ay) with a sampling frequency of $100 \mathrm{~Hz}$. Details on the motor imagery experiments performed by the subjects are given in [12]. As indicated in [12], the visual cues for the two motor imageries the subject should perform: Right hand (Class 1) and Right Foot (Class 2) are time-locked within 3.5 seconds. In this study, the signals obtained from C3 and $\mathrm{C} 4$ electrodes are filtered using Laplace Filters [12] to reduce the effects from the neighboring electrodes.

\subsection{Dataset II: Experimental Data}

This dataset was constructed from the EEG acquired from nine subjects during a leftright motor imagery experiment conducted at our lab. EEG signals were acquired using a 19 channel NeuroWin (manufactured by NASAN) amplifier, with a sampling frequency of $250 \mathrm{~Hz}$. Signals from C3 and C4 electrodes were selected to extract relevant information on the different movement. Prior to the start of the experiment, the subjects were given a small introduction about the research work and stages of the experiment involved. The subjects performed the experiments on a single day, consisting of 3 separate sessions with fifteen minutes relaxation in between.

The subjects perform the motor imagery tasks based on the visual stimuli shown to them during each experimental session. Each session comprises 30 trials, thus for every subject a total of 90 trials are obtained. The subjects imagine moving their right and the left hand when a right and left arrow is displayed on screen. In each session, a blank screen was displayed in the first 10 seconds. In the 10th second a fixation cross "+" was displayed on the screen which indicates the beginning of a trial. From the 12th second onwards, the visual stimuli is displayed for three seconds to indicate which arm to move. Next, a blank screen is shown for 2 seconds during which the subject can relax. This stage also reduces the effect of the previous motor imagery performed by the subject on the current one. Fig. 3 gives a generic structure of the visual stimuli.

Figure 3.

First, the acquired EEG is band-pass filtered between 8 to $35 \mathrm{~Hz}$ using an IIR elliptical filter of order 14, to remove the noise acquired from the amplifier and the environment. The elliptical was selected because the filter has a sharper roll off as compared to the other filters, requires a small filter order and can independently adjust both the passband and stopband ripples, as per the user's wish. The passband and stopband attenuation is expermentally determined to be $1 \mathrm{~dB}$ and $50 \mathrm{~dB}$, respectively. Then from each trial, the average of the two seconds of data acquired during the fixation cross period is subtracted from the three seconds of the movement stimuli data to remove the effect of background 
EEG from the motor imagery data. Finally, three seconds of the motor imagery data from each trial is selected for feature extraction.

\subsection{Feature Extraction: Power Spectral Density Estimates}

Spectrum estimation describes the power distribution contained in a signal over frequency based on a finite set of data. For our study, we have used the Welch's Periodogram for the spectral estimation of the EEG data. Here, the data segments are overlapped and windowed prior to the calculation of the periodogram. The overlapping and windowing of the data segments leads to a decrease in the variance and more control over the bias/resolution properties of the calculated PSD, respectively. These characteristics make this method highly suitable for analysis of a non-stationary signal [5].

For our study, we have prepared the original feature vector from PSD etimates using the welch's method. Here, a hamming window of size 125 and 50 for dataset I and II, respectively and 50\% overlap were used to obtain the frequency distribution of the extracted filtered EEG over 128 frequency points, for both the electrodes $\mathrm{C} 3$ and $\mathrm{C} 4$. Thus, the total size of the feature vector is 128 features $\times 2$ electrodes.

\subsection{Feature Selection and Classification Results}

The features extracted from the previous section is fed as inputs to our proposed feature selection. According to our algorithm, the best minimum number of features are selected which would yield the best result, i.e., classification accuracy. Thus, the classifiers used in this study have two functions: First, it is used to optimize the LA-DE feature selection and secondly, to validate the selection of the features on a test dataset. For this purpose, we have selected Support Vector Machines (SVM) [36, 42] with linear kernel as the classifier. SVM has earned popularity in recent years because it has good recognition ability at high computational speed as compared to other standard classifiers. Experiments undertaken further reveals that the parameters in the LA-DE algorithm which gives the best performance are as follows: Population Size $=50$, Scaling Factor $=0.5$, Crossover Ratio $=0.9$, maximum number of generations $=$ 10000 , stopping criteria $=10$ number of same fitness and Reward/Penalty Rate $=$ 0.01 . Table 1 and 2 gives the recognition accuracy of the classifiers before and after feature selection for both dataset I and II, respectively. For this purpose, the dataset is partitioned into training set and test set using k-fold cross validation technique. Here, $\mathrm{k}$ is selected as 10 and the accuracies for each subject is 
measured over 10 runs. As observed from Table 1 and 2, the accuracies of the dataset after employing the LA-DE algorithm have significantly improved.

Table 1.

Table 2 .

\section{Performance Analysis}

In this section, we examine the performance of the LA-DE algorithm with the following competitor algorithms: Particle Swarm Optimatization (PSO) [18], Genetic Algorithm (GA) [8] and Kernelized Principal Component Analysis (kPCA) [4] using linear SVM as the classifier. The parameters selected for PSO and GA are similar to the one selected for LA-DE in section 3.4. For kPCA we have reduced the dimensions of the algorithm to half of its original length, i.e., 128. The performance of the algorithms are measured by the following parameters: i)recognition accuracy (Acc.), ii) computational time (C.T) and features selected (F.S). They are defined below for ready reference.

Recognition Accuracy (Acc.): The ratio of the number of test data correctly classified by the trained classifier to the total number of test data. It is expressed in percentage (\%). Computational Time (C.T): The total time taken by the feature selection algorithm to produce the best result. It is expressed in seconds $(\mathrm{sec})$.

Features Selected (F.S): The number of features selected after using the feature-selection algorithms on the original datasets.

Each performance measures were calculated on MATLAB 7.9 environment run on a computer with the following specifications: Intel Core 2 Duo $1.19 \mathrm{GHz}, 3.2 \mathrm{~GB}$ RAM and Windows platform. Table 3 and 4 provides the results for the comparison of LA-DE with its competitors. As observed from Table 3, LA-DE yields the best result in terms of accuracy but PSO gives the best result in terms of features selected. But from Table 4 it is observed that LA-DE yields the best performance in terms of both accuracy and features selected. From both the table it is noted that kPCA requires the minimum amount of time but the accuracy obtained is very poor. Maintaining a trade-off between the accuracy, computational time and features selected, it is noted from Table 3 and 4 that LA-DE gives the best optimal result.

Table 3 .

Table 4.

The performance measures of LA-DE is further validated by means of Friedman's Test $[10,11]$ performed on both datasets I and II. The null hypothesis here, states that all the algorithms are equivalent, so their ranks $R_{m}$ should be equal. The Friedman statistic, 


$$
\chi_{F}^{2}=\frac{12 N}{k(k+1)}\left[\sum_{m=1}^{k} R_{m}^{2}-\frac{k(k+1)^{2}}{4}\right]
$$

is distributed accordingly to $\chi_{F}^{2}$ with $k-1$ degrees of freedom, where $k$ is the number of algorithms to be compared and $N$ is the number of parameters used for comparison. In this study, we have selected the mean of all the three parameters for both the dataset, thus, $k=4$ and $N=6$ and Table 5 is the ranking table prepared from Table 3 and 4.

Table 5 .

From Table 5 we calculate the value of $R_{j}$, which is further used in eqn (14) to get $\chi_{F}^{2}=$ $22.5>\chi_{4,0.095}^{2}=9.488$. So, the null hypothesis, claiming that all the algorithms are equivalent, is wrong and, therefore, the performances of the algorithms are determined by their ranks only. It is clear from the table that the rank of LA-DE is 1.5, claiming LA-DE yields better results than its competitors.

\section{Conclusion}

The paper proposes a novel feature selection technique based on Differential Evolution and Learning Automata. Experiments have been performed on two datasets using Power Spectral Density for features extraction and SVM as the pattern classifier. Comparisons have been performed with GA, PSO and kPCA and the results indicate that the proposed approach yields better result. A major advantage of the proposed algorithm is that the optimal number of relevant features is a direct outcome of the algorithm and does not have to be experimentally determined, which saves time of the user. Also, this algorithm allows the user to employ other classifiers in place of the ones discussed in this paper. The only disadvantage of this algorithm is that the performance of the optimizer may degrade due to stagnation. However, this can be easily overcome by running the optimizer a number of times (say 10) for the same cost function and then taking the best results. This does not affect the computational time as the optimizer works in an offline environment. Further study in this direction aims to optimize the feature extraction and classification techniques to be implemented in the online classification of the EEG data for BCI research, and thus to ultimately develop a complete stand-alone system for an EEG driven neuro-prosthetic control for rehabilitation purpose.

\section{Acknowledgements.}

I would like to thank University Grants Commission, India, University of Potential Excellence Programme (Phase II) in Cognitive Science, Jadavpur University and Council of Scientific and Industrial Research, India. 


\section{Reference}

1. Abdi H, Williams LJ (2010) Principal component analysis. Wiley Interdisciplinary Reviews: Computational Statistics 2:433-459

2. Alpaydin E (2004) Introduction to Machine Learning (Adaptive Computation and Machine Learning). The MIT Press

3. Birbaumer N, Murguialday AR, Cohen L (2008) Brain-computer interface in paralysis. Curr Opin Neurol 21 (6):634-638

4. Cao LJ, Chua KS, Chong WK, Lee HP, Gu QM (2003) A comparison of PCA, KPCA and ICA for dimensionality reduction in support vector machine. Neurocomputing 55 (1-2):321-336

5. Childers DG (1978) Modern spectrum analysis. IEEE Press

6. Chou C-H, Su M-C, Lai E (2004) A new cluster validity measure and its application to image compression. Pattern Anal Appl 7 (2):205-220

7. Comon P (1994) Independent component analysis, a new concept? Signal Process 36 (3):287314

8. Corralejo R, Hornero R, Alvarez D (2011) Feature selection using a genetic algorithm in a motor imagery-based Brain Computer Interface. In: Proceedings of the 2011 Annual International Conference of the IEEE Engineering in Medicine and Biology Society, EMBC, Boston, MA, pp 7703-7706. DOI 10.1109/IEMBS.2011.6091898

9. Das S, Abraham A, Konar A (2008) Automatic Clustering Using an Improved Differential Evolution Algorithm. IEEE Trans. SMC Part A: Systems and Humans 38 (1):218-237

10. Demšar J (2006) Statistical comparisons of classifiers over multiple data sets. J. Mach. Learn. Res. 7:1-30

11. Dietterich TG (1998) Approximate statistical tests for comparing supervised classification learning algorithms. Neural Comput. 10 (7):1895-1923

12. Dornhege G (2007) Toward Brain-Computer Interfacing. MIT Press

13. Dornhege G, Blankertz B, Curio G, Muller K (2004) Boosting bit rates in noninvasive EEG single-trial classifications by feature combination and multiclass paradigms. IEEE Trans. Biomed. Eng. 51 (6):993-1002

14. Dyson M, Sepulveda F, Gan J.Q (2008) Mental task classification against the idle state: A preliminary investigation. In: Proceedings of the 30th Annual International Conference of the IEEE Engineering in Medicine and Biology Society, Vancouver, BC, pp 4473-4477. DOI 10.1109/IEMBS.2008.4650206

15. Finke A, Knoblauch A, Koesling H, Ritter H (2011) A hybrid brain interface for a humanoid robot assistant. In: Proceedings of the 2011 Annual International Conference of the IEEE Engineering in Medicine and Biology Society, Boston, MA, pp 7421-7424. DOI 10.1109/IEMBS.2011.6091728

16. Galan F, Nuttin M, Lew E, Ferrez PW, Vanacker G, Philips J, Millan Jdel R (2008) A brainactuated wheelchair: asynchronous and non-invasive Brain-computer interfaces for continuous control of robots. Clin Neurophysiol 119 (9):2159-2169

17. Hansen PC (1986) The truncated SVD as a method for regularization. Stanford University 
18. Hu Z, Chen G, Chen C, Xu H, Zhang J (2010) A new EEG feature selection method for selfpaced brain-computer interface. In: Proceedings of the 10th International Conference on Intelligent Systems Design and Applications, Cairo. DOI 10.1109/ISDA.2010.5687156

19. J.C. T, Comair YG (2006) Central Region and Motor Cortex. In: Atlas of Regional Anatomy of the Brain Using MRI. Springer Berlin Heidelberg, pp 117-138

20. Kauhanen L, Jylanki P, Lehtonen J, Rantanen P, Alaranta H, Sams M (2007) EEG-based brain-computer interface for tetraplegics. Intell Neuroscience 2007:1-11

21. Lin H, Zhuliang Y, Zhenghui G, Yuanqing Li (2009) Bhattacharyya bound based channel selection for classification of motor imageries in EEG signals. In Proceedings of the Chinese Control and Decision Conference, Guilin, pp 2353-2356. DOI 10.1109/CCDC.2009.5192711

22. Lotte F, Congedo M, Lecuyer A, Lamarche F, Arnaldi B (2007) A review of classification algorithms for EEG-based brain-computer interfaces. J Neural Eng 4 (2):R1-R13

23. Mason SG and Birch GE (2003) A General Framework for Brain-Computer Interface Design. IEEE Trans Neural Sys. \& Rehab. Eng. 11(1): 70-85

24. Michele MT, Robert RL, Rudiger RR, Jose Del RJRM (2010) Towards natural non-invasive hand neuroprostheses for daily living. In: Proceedings of the 2010 Annual International Conference of the IEEE Engineering in Medicine and Biology Society, Beunos Aires, pp 126129. DOI 10.1109/IEMBS.2010.5627178

25. Mueller-Putz G, Scherer R, Pfurtscheller G, Neuper C (2010) Temporal coding of brain patterns for direct limb control in humans. Frontiers in Neuroscience 4(34)

26. Narendra KS, Thathachar M (1974) Learning Automata - A Survey. IEEE Trans SMC SMC4(4): 323-334

27. Nijholt A, Tan D (2008) Brain-Computer Interfacing for Intelligent Systems. IEEE Intel. Sys. $23(3): 72-79$

28. Pfurtscheller G, Neuper C, Muller GR, Obermaier B, Krausz G, Schlogl A, Scherer R, Graimann B, Keinrath C, Skliris D, Wortz M, Supp G, Schrank C (2003) Graz-BCI: state of the art and clinical applications. IEEE Trans Neural Sys. \& Rehab. Eng. 11 (2):1-4

29. Pfurtscheller G, Neuper C, Schlogl A, Lugger K (1998) Separability of EEG signals recorded during right and left motor imagery using adaptive autoregressive parameters. IEEE Trans Rehab. Eng. 6 (3):316-325

30. Prasad G, Herman P, Coyle D, McDonough S, Crosbie J (2010) Applying a brain-computer interface to support motor imagery practice in people with stroke for upper limb recovery: a feasibility study. J NeuroEng. Rehabil 7 (1):1-17

31. Price K, Storn RM, Lampinen JA (2005) Differential Evolution: A Practical Approach to Global Optimization (Natural Computing Series). Springer-Verlag New York, Inc

32. Rakotomamonjy A, Guigue V, Mallet G, Alvarado V (2005) Ensemble of SVMs for improving brain computer interface p300 speller performances. In: Duch W, Kacprzyk J, Oja E, Zadrozny S (eds) 15th international conference on Artificial Neural Networks: Biological Inspirations (ICANN), LNCS 3696, Springer, Berlin Heidelberg, pp 45-50. DOI 10.1007/11550822_8

33. Sanei S, Chambers JA (2008) EEG Signal Processing. Wiley 
34. Scholkopf B, Smola A, Muller K-R, (1998) Nonlinear component analysis as a kernel eigenvalue problem. Neural Comput 10 (5):1299-1319

35. Sengupta A, Chakraborti T, Konar A, Eunjin K, Nagar AK (2012) An Adaptive Memetic Algorithm using a synergy of Differential Evolution and Learning Automata. In: Proceedings of the 2012 IEEE Congress on Evolutionary Computation (CEC), Brisbane, QLD, pp 1-8. DOI 10.1109/CEC.2012.6256574

36. Shawe-Taylor J, Sun S (2011) A review of optimization methodologies in support vector machines. Neurocomputing 74(17): 3609-3618

37. Storn R, Price K (1997) Differential Evolution - A Simple and Efficient Heuristic for Global Optimization over Continuous Spaces. J of Global Optimization 11 (4):341-359

38. Sun S (2010) Extreme energy difference for feature extraction of EEG signals. Expert Sys. With App. 37(6): 4350-4357

39. Sun S (2008) The extreme energy ratio criterion for EEG feature extraction. In: Kurkova V, Neruda R, Koutnik J (eds) International Conference on Artificial Neural Networks (ICANN), LNCS 5164, Springer, Berlin Heifelberg, pp 919-928. DOI 10.1007/978-3-540-87559-8_95 40. Sun S, Zhang C (2006) Adaptive Feature Extraction for EEG Signal Classification, J. Med. \& Bio. Eng. \& Com. 44(10): 931-935

41. Theodoridis S, Koutroumbas K (2006) Pattern Recognition. Elsevier Science

42. Vapnik VN (1995) The nature of statistical learning theory. Springer-Verlag New York, Inc.

43. Yew-Soon O, Meng-Hiot L, Xianshun C (2010) Memetic Computation-Past, Present \& Future. IEEE Computational Intelligence Magazine 5 (2):24-31

44. Yongwook C, Jaeseung J, Sungho J (2012) Toward Brain-Actuated Humanoid Robots: Asynchronous Direct Control Using an EEG-Based BCI. IEEE Trans. Robotics 28 (5):11311144 


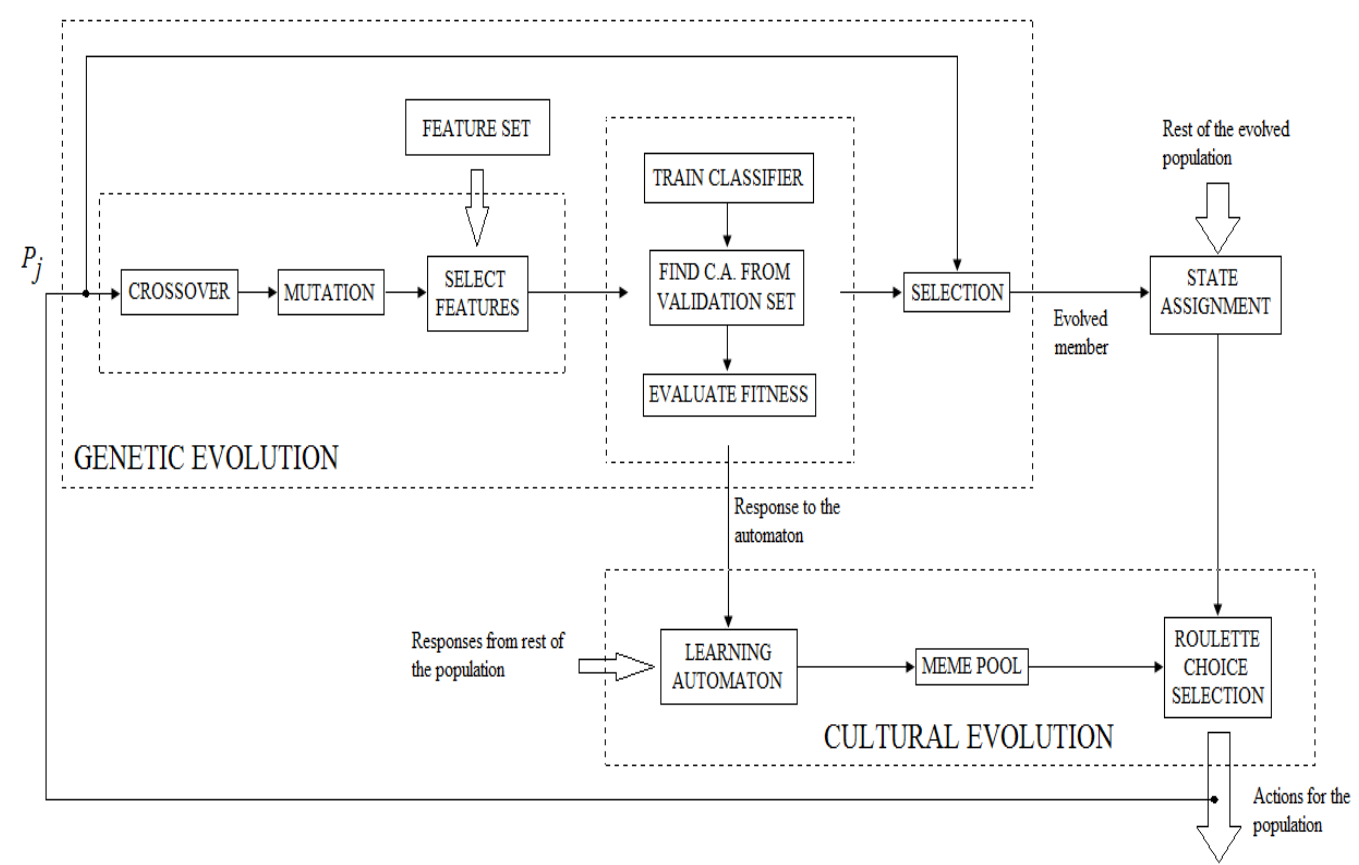

Figure 1: The proposed feature selection scheme. 


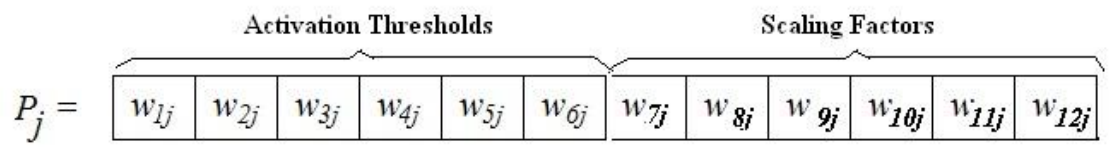

$$
\begin{aligned}
& \begin{array}{|l|l|l|l|l|l|l|l|l|l|l|l|}
\hline \mathbf{0} .64 & 0.72 & 0.93 & 0.46 & 0.34 & 0.23 & 0.68 & 0.76 & 0.44 & 0.90 & 0.21 & 0.33 \\
\hline
\end{array}
\end{aligned}
$$

Figure 2: An example of the vector representation of a particular population member for a dataset with 6 features. 


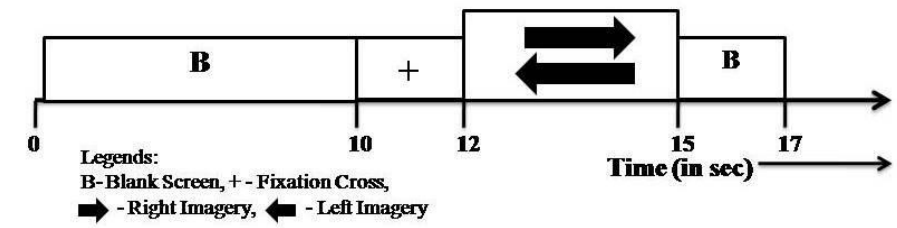

Figure 3: Timing Scheme Diagram of the Visual Cue. 
Table 1: Classification Accuracies (in \%) for dataset I (S.D.- Standard Deviation)

\begin{tabular}{llllllll}
\hline Subjects & aa & al & av & aw & ay & Mean & S.D. \\
\hline SVM & 88.24 & 95.45 & 77.78 & 100 & 100 & 92.29 & 9.43 \\
LA-DE-SVM & 97.06 & 100 & 100 & 100 & 100 & 99.41 & 1.31 \\
\hline
\end{tabular}


Table 2: Classification Accuracies (in \%) for dataset II (S.D.- Standard Deviation)

\begin{tabular}{llllllllllll}
\hline Subjects & 1 & 2 & 3 & 4 & 5 & 6 & 7 & 8 & 9 & Mean & S.D. \\
\hline SVM & 71.63 & 62.80 & 67.12 & 54.55 & 49.89 & 62.16 & 66.87 & 69.99 & 34.10 & 59.90 & 11.98 \\
LA-DE- & 100 & 88.89 & 87.50 & 88.89 & 77.78 & 88.89 & 100 & 90.00 & 70.00 & 87.99 & 9.51 \\
SVM & & & & & & & & & & & \\
\hline
\end{tabular}


Table 3. Comparison of the performance measures of LA-DE with other algorithms for Dataset I

\begin{tabular}{cccccccccccccc}
\hline & \multicolumn{3}{c}{ LA-DE } & \multicolumn{3}{c}{ PSO } & \multicolumn{3}{c}{ GA } & \multicolumn{3}{c}{ kPCA } \\
\cline { 2 - 12 } & Acc. & C.T. & F.S. & Acc. & C.T. & F.S. & Acc. & C.T. & F.S. & Acc. & C.T. & F.S. \\
\hline aa & 97.06 & 312 & 134 & 94.11 & 454 & 112 & 90.29 & 512 & 134 & 82.35 & 121 & 128 \\
al & 100 & 305 & 119 & 100 & 472 & 125 & 95.55 & 520 & 145 & 97.78 & 120 & 128 \\
av & 100 & 325 & 131 & 94.45 & 472 & 123 & 92.09 & 534 & 156 & 66.67 & 120 & 128 \\
aw & 100 & 305 & 117 & 100 & 472 & 122 & 100 & 520 & 144 & 100 & 121 & 128 \\
ay & 100 & 311 & 128 & 100 & 472 & 120 & 100 & 509 & 130 & 100 & 120 & 128 \\
\hline \multirow{2}{*}{ Mean } & $\mathbf{9 9 . 4 1}$ & $\mathbf{3 1 1 . 6}$ & $\mathbf{1 2 6}$ & $\mathbf{9 7 . 7 1}$ & $\mathbf{4 6 8 . 4}$ & $\mathbf{1 2 0}$ & $\mathbf{9 5 . 5 9}$ & $\mathbf{5 1 9}$ & $\mathbf{1 4 2}$ & $\mathbf{8 9 . 3 6}$ & $\mathbf{1 2 0 . 4}$ & $\mathbf{1 2 8}$ \\
\hline
\end{tabular}


Table 4. Comparison of the performance measures of LA-DE with other algorithms for Dataset II

\begin{tabular}{ccccccccccccccc}
\hline \multirow{2}{*}{ Subjects } & \multicolumn{3}{c}{ LA-DE } & \multicolumn{4}{c}{ PSO } & \multicolumn{3}{c}{ GA } & \multicolumn{3}{c}{ kPCA } \\
\cline { 2 - 5 } & Acc. & C.T. & F.S & Acc. & C.T & F.S & Acc. & C.T. & F.S & Acc. & C.T & F.S \\
\hline 1 & 100 & 234 & 129 & 100 & 344 & 127 & 99.8 & 370 & 130 & 78.89 & 75 & 128 \\
2 & 88.89 & 225 & 123 & 87.50 & 350 & 132 & 85.55 & 370 & 130 & 60.00 & 76 & 128 \\
3 & 87.50 & 234 & 134 & 75.00 & 350 & 130 & 80.00 & 378 & 135 & 70.00 & 75 & 128 \\
4 & 88.89 & 219 & 114 & 80.00 & 344 & 127 & 80.00 & 376 & 134 & 65.12 & 75 & 128 \\
5 & 77.78 & 220 & 119 & 77.78 & 356 & 138 & 77.78 & 378 & 135 & 66.67 & 77 & 128 \\
6 & 88.89 & 220 & 121 & 87.50 & 360 & 143 & 85.00 & 379 & 137 & 66.67 & 75 & 128 \\
7 & 100 & 226 & 127 & 88.89 & 321 & 109 & 90.00 & 361 & 121 & 72.22 & 74 & 128 \\
8 & 90.00 & 225 & 123 & 88.89 & 344 & 127 & 83.33 & 384 & 143 & 66.67 & 73 & 128 \\
9 & 70.00 & 228 & 126 & 66.67 & 363 & 152 & 60.00 & 390 & 150 & 60.90 & 75 & 128 \\
\hline Mean & $\mathbf{8 7 . 9 9}$ & $\mathbf{2 2 5 . 6 7}$ & $\mathbf{1 2 4}$ & $\mathbf{8 3 . 5 8}$ & $\mathbf{3 4 8}$ & $\mathbf{1 3 2}$ & $\mathbf{8 2 . 3 8}$ & $\mathbf{3 7 6 . 2 2}$ & $\mathbf{1 3 5}$ & $\mathbf{6 7 . 4 6}$ & $\mathbf{7 5}$ & $\mathbf{1 2 8}$ \\
\hline
\end{tabular}


Table 5. Ranking Table for performance comparison using Friedman's Test

\begin{tabular}{llllll}
\hline & & LA-DE & PSO & GA & kPCA \\
\hline Dataset I & Acc & 1 & 2 & 3 & 4 \\
& C.T & 2 & 3 & 4 & 1 \\
& F.S & 2 & 1 & 4 & 3 \\
\hline Dataset II & Acc & 1 & 2 & 3 & 4 \\
& C.T & 2 & 3 & 4 & 1 \\
& F.S & 1 & 2 & 4 & 3 \\
\hline Avg. Rank & & 1.5 & 2 & 4 & 3 \\
\hline
\end{tabular}

